\title{
Effects of 5-HT 4 selective receptor agonist, mosapride citrate on electrocardiogram in dogs
}

\author{
Ji Sang Chae, Jin Ok Ahn, Ye Rin Coh, Chong Woo Park, Hwa Young Youn* \\ College of Veterinary Medicine, Seoul National University, Seoul 151-742, Korea \\ (Received: October 5, 2011; Revised: July 5, 2012; Accepted: July 6, 2012)
}

\begin{abstract}
Mosapride stimulated dietary motility was introduced because of the arrhythmogenic effect of cisapride. Cisapride, 5-HT receptor agonist, induces prolongation of QT interval. Additionally, this condition can raise the possibility of acute, "malignant" arrhythmias such as torsade de pointes. It is hard to find any reports about effects of mosapride on cardiac parameters in dogs. By confirming electrocardiogram (ECG) parameters, the surface extremity leads ECG that was obtained from the four-limb electrodes and which was recorded by an ECG recorder after administration of mosapride $3 \mathrm{mg} / \mathrm{kg}$ PO b.i.d, and mosapride $3 \mathrm{mg} / \mathrm{kg}$ with itraconazole $5 \mathrm{mg} / \mathrm{kg}$ PO b.i.d, respectively. QT interval was shortened on the days of 3,5 , and post-day 1 in both mosapride $3 \mathrm{mg} / \mathrm{kg}$ administrated group and mosapride with itraconazole group. Heart rate increased significantly. QTc was slightly prolonged in mosapride administration group and mosapride with itraconazole group. However, all dogs of QTc were in normal variation (150 250 msec). Besides, the dogs showed no side effects reported in human medicine during the administration with these drugs. Although mosapride can increase the heart rate, this study suggest that mosapride may be useful for the dogs with disorders of gastrointestinal motility because of no fatal arrhythmogenic effect inspite of administration with itraconazole in dogs.
\end{abstract}

Keywords : electrocardiogram, itraconazole, mosapride, QTc, QT interval

\section{Introduction}

The greatest scientific and clinical advances in gastroenterology over the past two decades have addressed the functional aspect of digestion, especially in gastrointestinal motility. With the smooth muscle contraction of gastrointestinal system, peristalsis tends to propel digestive contents along the gastrointestinal tracts. Delayed gastric emptying is a significant cause of upper gastrointestinal tract symptoms in dogs and cats [4].

Some gastrointestinal prokinetic agents have effects throughout the gastrointestinal tract. The gastrointestinal prokinetic agents work with many different mechanisms of action [2]. Prokinetic agents, including metoclopramide, cisapride and mosapride increase digestive motility by, stimulating serotonin $5-\mathrm{HT}_{4}$ receptors in the gastrointestinal plexus, consequently increasing the release of acetylcholine and enhancing gastrointestinal motility as well as gastric emptying [2]. Metoclopramide stimulates upper gastrointestinal tract than colonic motility that has more potent as antiemetic than upper gastrointestinal motility [7,9]. Animals show unusual behavior, extrapyramidal side effect after administration rarely because this drug originally marketed as a dopamine transporter receptor antagonist $[7,8]$. Besides, metoclopramide is excreted mostly in the urine, and severe renal failure makes adverse effects more likely [8]. Cisapride is an oral gastrointestinal prokinetic agent that stimulates normal motility from the lower esophageal sphincter to the anus. It has about 8 times more potent than metoclopramide as a prokinetic agent does [12]. However, cisapride, which acts on serotonin receptors $5-\mathrm{HT}_{1}$ to $5-\mathrm{HT}_{4}$, has been associated with potentially fatal heart rhythm abnormalities [7]. Cisapride induces prolongation of QT interval. Furthermore, this condition can raise the possibility of such acute, "malignant" arrhythmias as torsade de pointes and ventricular fibrillation [13]. Concomitant use of imidazole class antifungal agent or macrolide antibiotics has also been found to inhibit the cytochrome P-450 enzyme system that affects cisapride metabolism and results in increased serum cisapride levels and more arrythmogenic [13]. For that reason, this drug has not been available from several markets including Korea [2].

Many alternative gastrointestinal prokinetic agents have been introduced in market like itopride, and mosapride which are a structurally related benzamide, because of arrhythmogenic effect of cisapride. Mosapride enhances upper gastrointestinal motility in conscious dogs $[6,14]$. In rabbits, rats, guinea pigs, and conscious cats, mosapride does not show arrhythmogenic effect $[1,5]$. Although mosapride can

*Corresponding author

Tel: +82-2-880-1266, Fax: +82-2-880-8682

E-mail: hyyoun@snu.ac.kr 
be possible to alternate the uses of cisapride and metoclopramide, there is little information about side effects of mosapride in dogs. There is no report about effects on cardiac parameters in dogs. This study is to investigate the cardiac effects of mosapride citrate on electrocardiogram (ECG) in dogs.

\section{Materials and Methods}

\section{Preparation of experimental animals}

The use of animals in this experiment was approved by the Institute of Laboratory Animal Resources, Seoul National University (SNU-110722-1), Korea. All the beagle dogs (6 dogs) were cared for in accordance with the Animal Care and Use Guidelines (Institute of Laboratory Animal Resources, Seoul National University). The animals used in our experiment were male or female beagle dogs weighing $6 \sim 10 \mathrm{~kg}$. All the dogs were determined to be healthy after a routine physical examination, complete blood count, serum biochemistry analysis. Each dog was kept in its cage at a room temperature (approximately $25^{\circ} \mathrm{C}$ ). Food was available twice a day and water was continuously available. The animals were divided into 2 groups: mosapride $3 \mathrm{mg} / \mathrm{kg}$ administration group (A group: $n=3,2$ males and 1 female), and mosapride $3 \mathrm{mg} / \mathrm{kg}$ with itraconazole $5 \mathrm{mg} / \mathrm{kg}$ group (B group: $\mathrm{n}=3,2$ males and 1 female). Dogs were orally and repeatedly administered for 5 days with water, mosapride $3 \mathrm{mg} / \mathrm{kg}$ or mosapride with itraconazole $5 \mathrm{mg} / \mathrm{kg}$ twice a day. From the 1 st to 5 th day of administration, drugs were administered twice a day (8 9 a.m. and 8 9 p.m.).

\section{ECG measurement} trodes and recorded by using an ECG recorder (Cardiofax

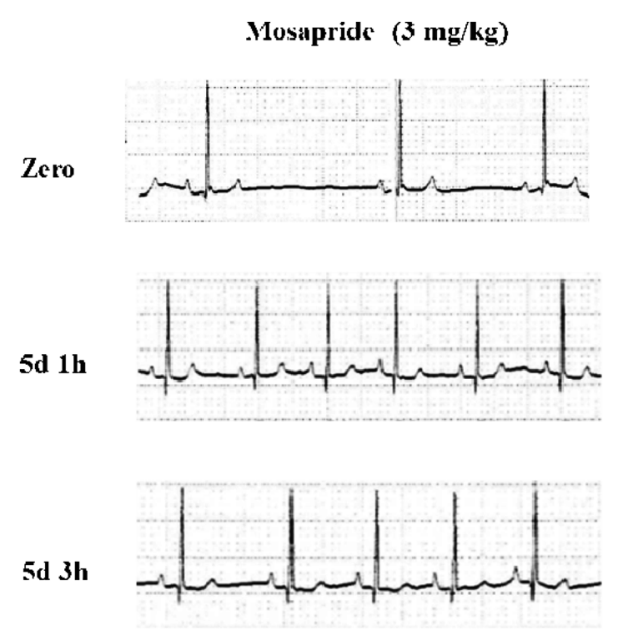

The surface ECG was obtained from the four-limb elec-

GEM ECG-9020K; Nihon Kohden, Japan). From the 1st to 5 th day of administration, ECG was measured on day 1, day 3 and day 5 at least 3 times at each point $(0,1$ and $3 \mathrm{~h}$ after administration). ECG was also evaluated post drug administration of 1 day and 2 days. Heart rate was measured before and after the administration of mosapride. ECG signals were read for $10 \sim 15 \mathrm{sec}$, and recorded on ECG paper. The signals were automatically analyzed by an interpretive ECG Analysis for dogs and cats (Veterinary ECG Software Kit QP992E; Nihon Kohden). We also checked the end of T wave and analyzed ECG manually, and then QT intervals were measured. The QT interval begins with the onset of the QRS complex and ends when the $T$ wave returns to baseline. Even though the QT interval does not vary during respiratory sinus arrhythmia, measured QT interval was corrected for the effect of heart rate using the Bazett formula: QTc $=$ QT inter$\mathrm{val} /$ square root of RR interval (in seconds).

\section{Drugs}

Mosapride was obtained from the commercial source (Gasmotin; Daewoong Pharmaceutical, Korea). Itraconazole was also obtained from the market (Hitrazole; JW Parmaceutical, Korea). Administration dose of mosapride was decided by motility index of recent study [6]. Dogs were orally administered mosapride or mosapride with itraconazole twice a day for 7 days.

\section{Statistical analysis}

All data are expressed as the mean \pm SD statistically significant differences between the values in drug-treated group were analyzed by One-Way ANOVA using the PASW 18 statistics (IBM, USA). All differences with values of $p \leq 0.05$ were considered significant.
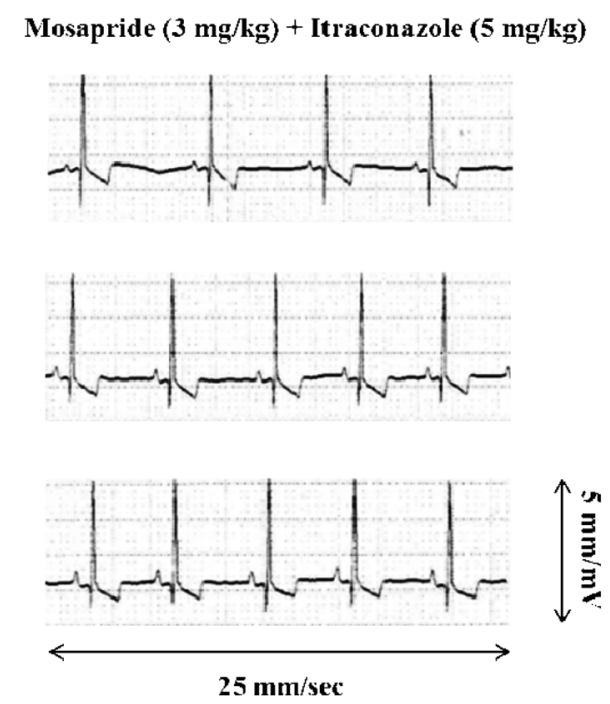

Fig. 1. Effects of mosapride on electrocardiogram at the 5 day (d) of repeated administration in conscious dogs. There are no fatal arrhythmogenic effects in mosapride $(3 \mathrm{mg} / \mathrm{kg})$ administration group and mosapride $(3 \mathrm{mg} / \mathrm{kg})$ with itraconazole $(5 \mathrm{mg} / \mathrm{kg})$ group. Lead as marked, $25 \mathrm{~mm} / \mathrm{sec}, 5 \mathrm{~mm} / \mathrm{mV}$. 


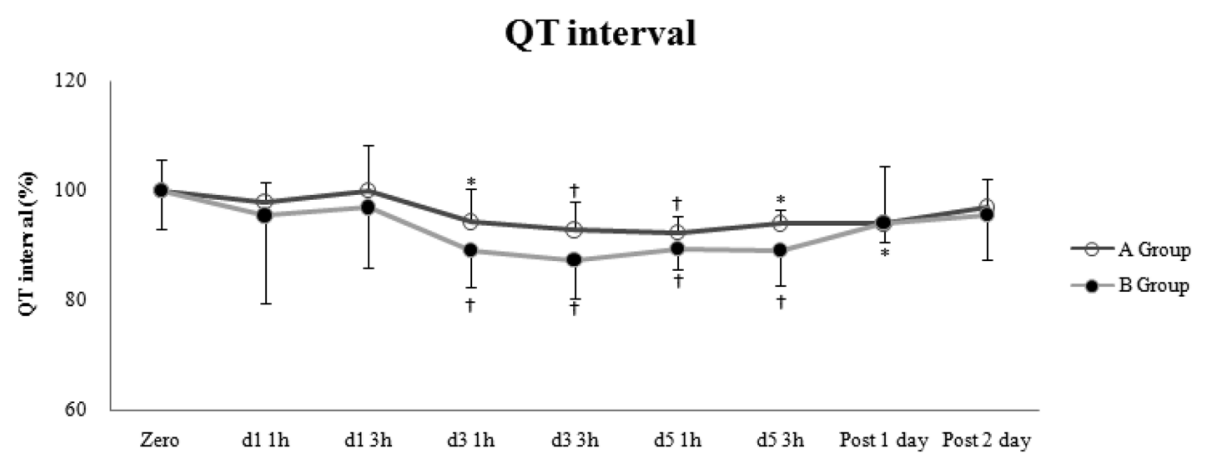

Fig. 2. QT intervals in $3 \mathrm{mg} / \mathrm{kg}$ mosapride administration group (A group), and $3 \mathrm{mg} / \mathrm{kg}$ mosapride with $5 \mathrm{mg} / \mathrm{kg}$ itraconazole administration group (B group). Compared with zero time (QT interval of zero time converted to 100), QT intervals of A group and B group were shortened significantly. In A group, QT interval was decreased on day (d) 3 (1 h: $94.2 \pm 6.0,3 \mathrm{h:} 92.7 \pm 5.4)$, d5 1 h (92.2 \pm 3.2 ) after drug administration, and post 1 day (94.0 \pm 10.4$)$. In B group, QT interval was decreased on d3 (1 h: 89.1 $\pm 6.8,3 \mathrm{~h}: 87.2 \pm 7.0)$, d5 (1 h: $89.3 \pm 3.7,3 \mathrm{~h}: 89.0 \pm 6.2$ ). There was not any significant difference between two groups of QT interval. Differences between zero time and the other day (mean $\pm \mathrm{SD}) .{ }^{*} p<0.05,{ }^{\dagger} p<0.01$.

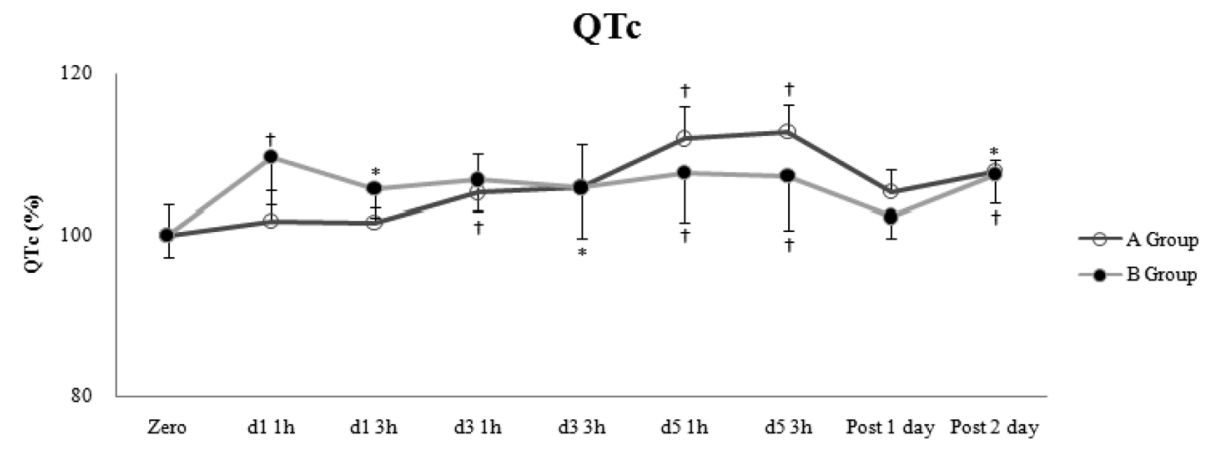

Fig. 3. QTc in $3 \mathrm{mg} / \mathrm{kg}$ mosapride administration group (A group), and $3 \mathrm{mg} / \mathrm{kg}$ mosapride with $5 \mathrm{mg} / \mathrm{kg}$ itraconazole administration group (B group). Compared with zero time (QTc of zero time converted to 100), QTc of A group and B group were slightly prolonged. In A group, QTe was prolonged on day (d) 5 ( $1 \mathrm{~h}: 111.8 \pm 4.2,3 \mathrm{~h}: 112.7 \pm 3.3)$ and post 2 days (107.8 \pm 1.4$)$. In B group, QTe was increased except post 1 day. QTc of d1 ( $1 \mathrm{~h}: 109.5 \pm 5.8,3 \mathrm{~h}: 105.7 \pm 3.6)$, d3 (1 h: $106.7 \pm 3.9,3 \mathrm{~h}: 105.7 \pm 6.1)$, d5 (1 h: $107.7 \pm$ $6.1,3 \mathrm{~h}: 107.2 \pm 6.6)$, and post 2 days $(107.9 \pm 3.6)$ were all increased significantly. There wasn't any significant difference between two groups of QTc. Differences between zero time and the other day (mean $\pm \mathrm{SD}$ ). ${ }^{*} p<0.05,{ }^{\dagger} p<0.01$.

\section{Results}

All groups did not have adverse effect such as diarrhea, loose stools, dry mouth, anorexia and vomiting which have been reported in human. Other laboratory abnormalities found in human clinical trials included eosinophilia, elevated triglycerides, and elevated alanine transaminase, alanine aminotransferase and gamma glutamyl transpeptidase levels. However, they did not show any abnormal laboratory test values.

A group did not affect on PR interval, $\mathrm{P}$ wave duration, $\mathrm{P}$ wave amplitude, QRS duration, QRS amplitude, ST segment, and had no arrhythmogenic effect (Fig. 1). However, mosapride had an effect on the QT interval or QTc in conscious dogs. QT interval was shortened on days of 3, 5, and post 1 day in both mosapride administrated group and mosapride with itraconazole group. But there was no statistical difference among three groups of QT interval (Fig. 2).
QTc was prolonged slightly in A group on the days of 5, and post 2 days. B group showed the effect on the QTc except post 1 day. However, there was no significant difference between two groups of QTc (Fig. 3). Heart rate increased on the days of $3(23.7 \pm 8.4 \%), 5(34.4 \pm 8.4 \%)$ in A group. In B group, heart rate was also increased on the day of $1(20.4 \pm$ $8.5 \%), 3(32.3 \pm 3.4 \%)$, and $5(33.4 \pm 9.0 \%)$ (Fig. 4).

\section{Discussion}

The cardiotoxic effect of cisapride derived from the ability of drug to prolong the cardiac action potential duration, which has been associated with the occurrence of prolonged QT interval followed by torsades de pointes [7, 13]. These effects are not mediated by $5-\mathrm{HT}_{4}$ receptor activation. Cisapride blocks human ether-a-go-go-related gene (HERG) $\mathrm{K}^{+}$channels that are responsible for the repolarization phase of cardiac action potential in human studies [2]. Although 


\section{Heart rate}
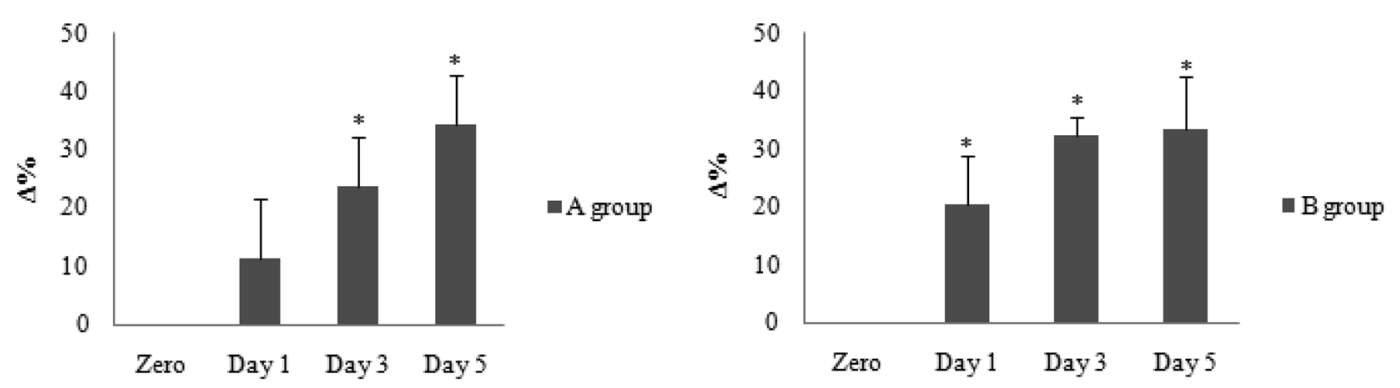

Fig. 4. Heart rate in $3 \mathrm{mg} / \mathrm{kg}$ mosapride administration group (A group), and $3 \mathrm{mg} / \mathrm{kg}$ mosapride with $5 \mathrm{mg} / \mathrm{kg}$ itraconazole administration group (B group). Compared with zero time (heart rate of zero time converted to 0), heart rate of A group and B group were increased. In A group, heart rate was increased on the days of $3(23.7 \pm 8.4 \%), 5(34.4 \pm 8.4 \%)$. In B group, heart rate was increased on the day of $1(20.4 \pm 8.5 \%), 3(32.3 \pm 3.4 \%)$, and $5(33.4 \pm 9.0 \%)$. Differences between zero time and the other day (mean \pm SD). " $p<0.05$.

other substituted benzamides like mosapride do not share Class III antiarrhythmic properties, cisapride has been known to have the pharmacophore for Class III antiarrhythmic agent [11]. Some human studies reported that mosapride did not change the parameters of autonomic nervous activity [3]. Even though mosapride did not have an effect of autonomic nervous activity in animals, it was reported that mosapride increased the heart rate significantly in dogs because of the 5- $\mathrm{HT}_{4}$ properties [3].

In the present study, heart rate increased significantly in dogs after the administration of $3 \mathrm{mg} / \mathrm{kg}$ of mosapride on the days of 3 , and 5 . In the dogs after the administration of mosapride with itraconazole, heart rate was elevated on the days of 1,3 , and 5 . Moreover, repeated oral administration of mosapride $3 \mathrm{mg} / \mathrm{kg}$ b.i.d and mosapride $3 \mathrm{mg} / \mathrm{kg}$ with itraconazole $5 \mathrm{mg} / \mathrm{kg}$ b.i.d for 7 days slightly shortened the QT interval in conscious dogs instead of showing prolonged QT interval. Increased heart rate is related to shortened RR interval even though QT interval does not vary during respiratory sinus arrhythmia. In the present of study, it seemed that QT interval was shortened significantly because of increased heart rate. After correction of QT interval using Bazett formula, QTc was slightly prolonged in mosapride administration group and mosapride with itraconazole administration group. All the dogs of QTc, however, are in normal variation (150 250 msec).

In human medicine, 56 percent of patients of taking medication of the imidazole class or macrolide antibiotics, which have been found to reduce the CYP450 enzyme, affect cisapride metabolism and raise serum cisapride levels. For that reason, these patients may have increased the risk of arrhythmia [13]. In the present study, there was no statistical difference between mosapride administration group and mosapride with itraconazole administration group of QT interval, QTc, and heart rate. Other study improved that mosapride has no arrhythmogenic effect although at serum concentration $30 \sim 100$ times in the cats showed higher than the therapeutic ones in man [5].

Most common adverse effects of mosapride were abdominal pain and diarrhea in human medicine. Abnormal laboratory test value reported eosinophilia, high liver enzyme, and elevated triglycerides [2]. In the present study, all the dogs do not show those side effects during the administration of these drugs. However, long-term use of mosapride should be done in order to verify whether this drug has significant side effects or not.

Although mosapride can increase the heart rate, this study suggest that mosapride may be useful for the dogs with disorders of gastrointestinal motility because of no fatal arrhythmogenic effect in spite of administration with itraconazole in dogs. The limitation of this study reveals small dose of mosapride, and short duration of administration. Also, we cannot demonstrate the dose-dependency side effects of mosapride on this study. In veterinary medicine, mosapride may be useful for treatment of gastrointestinal dysfunction in horse when mosapride has been administrated $2 \mathrm{mg} / \mathrm{kg}$ orally [10]. But further study will be needed to substitute mosapride established gastroprokinetic agents like cisapride and metoclopramide in dogs.

\section{Acknowledgments}

This work was supported by the Brain Korea 21 Program and the Research Institute for Veterinary Science, College of Veterinary Medicine, Seoul National University, Korea.

\section{Reference}

1. Carlsson L, Amos GJ, Andersson B, Drews L, Duker G, Wadstedt G. Electrophysiological characterization of the prokinetic agents cisapride and mosapride in vivo and in vitro: implications for proarrhythmic potential? J Pharmacol Exp Ther 1997, 282, 220-227. 
2. Curran MP, Robinson DM. Mosapride in gastrointestinal disorders. Drugs 2008, 68, 981-991.

3. Endo $\mathbf{J}$, Nomura $M$, Morishita $\mathbf{S}$, Uemura $\mathbf{N}$, Inoue $\mathbf{S}$, Kishi S, Kawaguchi R, Iga A, Ito S, Nakaya Y. Influence of mosapride citrate on gastric motility and autonomic nervous function: evaluation by spectral analyses of heart rate and blood pressure variabilities, and by electrogastrography. J Gastroenterol 2002, 37, 888-895.

4. Hall JA, Washabau RJ. Gastric prokinetic agents. In: Kirk RW, Bonagura JD (eds.). Kirk's Current Veterinary Therapy XIII. 13th ed. pp. 614-617, WB Saunders, Philadelphia, 2000.

5. Kii Y, Nakatsuji K, Nose I, Yabuuchi M, Mizuki Y, Ito T. Effects of 5-HT(4) receptor agonists, cisapride and mosapride citrate on electrocardiogram in anaesthetized rats and guinea-pigs and conscious cats. Pharmacol Toxicol 2001, 89, 96-103.

6. Matsunaga Y, Tanaka T, Yoshinaga K, Ueki S, Hori Y, Eta R, Kawabata Y, Yoshii K, Yoshida K, Matsumura T, Furuta S, Takei M, Tack J, Itoh Z. Acotiamide hydrochloride (Z-338), a new selective acetylcholinesterase inhibitor, enhances gastric motility without prolonging QT interval in dogs: comparison with cisapride, iltopride, and mosapride. J Pharmacol Exp Ther 2011, 336, 791-800.

7. Mohammad-Zadeh LF, Moses L, Gwaltney-Brant SM. Serotonin: a review. J Vet Pharmacol Ther 2008, 31, 187199.

8. Nelson RW, Couto CG. Small Animal Internal Medicine. 4th ed. pp. 404-406, Mosby, St. Louis, 2009.

9. Plumb DC. Plumb's Veterinary Drug Handbook. 6th ed. pp. 814-816, Wiley-Blackwell, Stockholm, 2008.

10. Sasaki N, Lee I, Ayukawa Y, Yamada H. Effects of orally administrated mosapride, a $5-\mathrm{HT}_{4}$ receptor agonist on the digestive tract of horses. J Equine Sci 2004, 15, 81-84.

11. Tonini M, De Ponti F, Di Nucci A, Crema F. Review article: cardiac adverse effects of gastrointestinal prokinetics. Aliment Pharmacol Ther 1999, 13, 1585-1591.

12. Webster CRL. Clinical Pharmacology. 1st ed. pp. 106-107, Teton NewMedia, Jackson, 2001.

13. Wysowski DK, Bacsanyi J. Cisapride and fatal arrhythmia. N Engl J Med 1996, 335, 290-291.

14. Yoshida N, Ito T, Karasawa T, Itoh Z. AS-4370, a new gastrokinetic agent, enhances upper gastrointestinal motor activity in conscious dogs. J Pharmacol Exp Ther 1991, 257, 781-787. 